\title{
Interaktionen erkennen
}

\author{
Karin Pinter
}

Die Qualität der therapeutischen Beziehung gilt als zentraler Faktor für die Wirksamkeit psychotherapeutischer Arbeit. Aber was sind die Zutaten einer qualitätsvollen und wirkungsvollen Beziehung? Der Versuch, Qualitätsmerkmale konkret zu benennen, führt unweigerlich zur Frage der therapeutischen Kommunikation, der impliziten und auch expliziten. Empirische Forschungsbeiträge zur Erhellung dieser Fragen lassen die Bedeutung der Selbstbeobachtung und Selbstwahrnehmung, insbesondere von PsychotherapeutInnen in der Interaktion mit ihren KlientInnen wieder stärker in den Fokus rücken.

Michael B. Buchholz stellt gemeinsam mit KoAutorInnen (Alder, Brakemeier, Dittmann, Dreyer) die Konversationsanalyse an Hand einer fallspezifischen Betrachtung vor. Dieser Beitrag ist Teil einer größeren Berliner Studie zur Empathie-Darstellung in therapeutischen Konversationen, die mittels aufwändiger Transkripte erfasst werden. Die Autorinnen und Autoren regen eine Reihe von Fragen an, die recht präzise den „Nerv" psychotherapeutischer Herausforderungen treffen, wie etwa die Bedeutung der Wechselseitigkeit und der Interaktion in der Psychotherapie, des "talking-in-interaction“; der therapeutischen Fehler und deren negative Effekte; das Thema von Dominanz und Unterwerfung im therapeutischen Kontext; des Einflusses von Forschungsmethoden (Fragebogen) auf den therapeutischen Prozess und der stets impliziten, aber selten explizit beantworteten Frage „Was tun wir hier?“.

Die Herausforderungen in der therapeutischen Arbeit mit einer schwer zugänglichen, vermeidend gebundenen Klientin beschreibt Karin Pinter in ihrem Beitrag vor dem theoretischen Hintergrund der Bindungsforschung und dem Konzept der Mentalisierung. Insbesondere die Regulation physiologischer Bedürfnisse wird in dieser Therapie im Zusammenhang mit Bindungsmotiven und der Mentalisierungsfähigkeit verstanden.

Um die Bedeutung von Interaktion in der ArztPatienten-Beziehung geht es in dem Beitrag von Melanie Esterl. Sie stellt den qualitativen Teil einer Studie vor, in der die Wahrnehmung der Arzt-Patient-Beziehung und deren Effekte sowohl durch die Ärztin als auch durch die Patientin untersucht werden. Die Patientinnen leiden an Endometriose, einer gynäkologischen, langdauernden und chronisch verlaufenden Krankheit.
K. Pinter $(\bowtie)$

Röntgengasse 7,

1170 Wien, Österreich

E-Mail: karin.pinter@wagnerpinter.at 